\title{
SYNTHESIS, CHARACTERIZATION AND THERMOGRAVIMETRIC
}

\section{STUDIES OF POLYMER RESIN DERIVED FROM 4-}

\section{HYDROXYACETOPHENONE AND CATECHOL}

\author{
C. S. Makde ${ }^{1}$, M. Ahamed ${ }^{1}$ and W. B. Gurnule ${ }^{2}$ \\ ${ }^{1}$ Department of Chemistry, Priyadarshini College of Engineering, Nagpur \\ 440019, Maharashtra, India. \\ 2Department of Chemistry, Kamla Nehru Mahavidyalaya, Sakkardara, \\ Nagpur 440024, Maharashtra, India \\ E-mail : wbgurnule@yahoo.co.in ,nwgurnule@gmail.com
}

\begin{abstract}
Polycondensation technique was employed to synthesize copolymer resins of 2,4dihydroxyacetophenone, adipamide, and formaldehyde in acidic medium with mole proportion of 1:1:2. The copolymer was characterized by ultraviolet, infra-red, nuclear magnetic resonance $\left({ }^{1} \mathrm{H}\right.$ and $\left.{ }^{13} \mathrm{C}\right)$ spectroscopy, scanning electron microscopy (SEM) and $\mathrm{X}$ - ray diffraction analysis (XRD). The thermal decomposition pattern and the kinetics of thermal decomposition of the copolymers were investigated by thermogravimetric analysis (TGA) in a static nitrogen atmosphere at a heating rate of $10^{\circ} \mathrm{C} / \mathrm{min}$. FreemanCarroll, Sharp- Wentworth, Freidman's, Chang and Coat Redfern methods have been adopted to evaluate the kinetic and thermodynamic parameters such as thermal activation energies $\left(E_{a}\right)$, order of the reaction $(n)$, entropy change $(\Delta S)$, free energy change $(\Delta \mathrm{F})$, apparent entropy $\left(\mathrm{S}^{*}\right)$, and frequency factor
\end{abstract}

Key words; TGA, Thermogravimetric analysis, Synthesis, polycondensation, Kinetic parameters

\section{INTRODUCTION}

The thermal degradation study of terpolymers has become a subject of interest. Study of thermal behavior of terpolymers in air at different temperature provides information about the nature of species produced at various temperatures due to degradation. Terpolymers having good thermal stability and catalytic activity have enhanced the development of polymeric materials. In this laboratory, extensive work on the thermal 
degradation of terpolymers has been undertaken [1-3]. It is hoped that the study of terpolymers will lead to the production of polymer, which are both thermally stable and useful as fabricating materials. Patel et al. [4] studied the thermal properties of copolymers and terpolymers. Zeman and Tokarova [5] prepared urea-formaldehyde copolymers and studied their TGA and DTA data.

Recently, we have reported the relative thermal stabilities of some new terpolymers derived from p-hydroxybenzaldehyde, biuret, and formaldehyde on the basis of their activation energies, calculated from non-isothermal TG data using the Sharp-Wentworth method as well as from isothermal TG data [6-7] widely used in the field of organic polymers. The tentative compositions and structures of the polymeric units have also been proposed. These display good agreement with the results of elemental analysis, electronic spectral, FTIR spectral, ${ }^{1} \mathrm{H}$ NMR and ${ }^{13} \mathrm{C}$ NMR spectral studies.

\section{MATERIALS AND METHODS}

\section{Synthesis of polymer 2,4-DHAAF}

The polymer resin (2,4-DHAAF) was synthesized by the condensation polymerization reaction of 2,4-dihydroxyacetophenone and adipamide with formaldehyde in 1:1:2 mole ratio by using hydrochloric acid as the reaction medium at $124 \pm 2{ }^{\circ} \mathrm{C}$ in an oil bath for $5 \mathrm{hrs}$ under refluxed condition with occasional shaking. The reaction mixture was then cooled. The dark brown colored polymer separated out was washed with warm water and extracted with diethyl ether. The dried sample was then purified by dissolving in $8 \%$ aqueous $\mathrm{NaOH}$ and regenerated using 1: $1(\mathrm{v} / \mathrm{v}) \mathrm{HCl} /$ water with constant and rapid stirring to avoid lump formation. The process of reprecipitation was repeated twice. The polymer 4-HACF-II thus obtained was filtered, washed with hot water, dried in air, powered and kept in vacuum desicator over silica gel. The yield of the polymer was found to be $85 \%$. 


\section{RESULTS AND DISCUSSION}

The synthesized and purified 2,4-DHAAF polymer resin was found to be dark brown in colour. The polymer is insoluble in commonly used solvent, but it is soluble in DMF, DMSO, pyridine and conc. $\mathrm{H}_{2} \mathrm{SO}_{4}$.

The UV-Visible spectra of the 2,4-DHAAF polymer sample in pure DMSO was recorded in the region 200-800 $\mathrm{nm}$ at a scanning rate of 100 $\mathrm{nm} \mathrm{m^{-1 }}$ and a chart speed of $5 \mathrm{~cm} \mathrm{~min}^{-1}$. The 2,4-DHAAF polymer sample gave two characteristics bands at 260-270nm and 205-215 $\mathrm{nm}$. These observed positions for the absorption bands have different intensities. The band at 270-280 $\mathrm{nm}$ more intense band which may be accounted for $\mathrm{a} \square \square *$ transition while the less intense band at 205-215 nm may be due to $\mathrm{n} \square$ * transition [19]. $\square \square$ transition indicates the presence of aromatic nuclei and $\mathrm{n} \square \square^{*}$ transition indicates presence of $\mathrm{OH}$ group. The hyperchromic effect is due to the presence of - $\mathrm{OH}$ group.

The infrared spectra of polymer 2,4-DHAAF resin. From the spectra it has been revealed that the polymer shows broad absorption band appeared at $3352 \mathrm{~cm}^{-1}$ may be assigned to the stretching vibrations of phenolic hydroxyl (-OH) groups exhibiting intramolecular hydrogen bonding [14]. A sharp strong peak at $1592 \mathrm{~cm}^{-1}$ may be ascribed to aromatic skeletal ring. The bands obtained at $1198 \mathrm{~cm}^{-1}$ suggest the presence of methylene $\left(-\mathrm{CH}_{2}\right)$ bridge [15]. The sharp and weak band obtained at $1279 \mathrm{~cm}^{-1}$ suggests the presence of $\mathrm{Ar}-\mathrm{CH}_{2}-\mathrm{Ar}$ bridge in polymer. The sharp band displayed at $1658 \mathrm{~cm}^{-1}$ may be due to stretching vibration of carbonyl group attached to acetophenone moity. The 1,2,3,4 substitution of aromatic benzene ring recognized by the sharp, medium / weak absorption bands appeared at 587, 825, 887, 977 and $1076 \mathrm{~cm}^{-1}$ respectively. This band seems to be merged with very broad band of phenolic hydroxyl group. 
The ${ }^{1}$ HNMR spectra of 2,4-DHAAF polymer was scanned in DMSO$\mathrm{d}_{6}$ solvent. The chemical shift ( $\partial$ ) ppm observed is assigned on the basis of data available in literature [16, 17]. From the spectral data, the singlet appearing at 3.56( $\partial$ ) ppm may be due to the methylene proton of $\mathrm{Ar}-\mathrm{CH}_{2}$ Ar moiety. The weak multiple signals (unsymmetrical pattern) in the region of $6.81(\partial) \mathrm{ppm}$ may be attributed to aromatic proton (Ar-H). The signals appearing at $8.21(\partial) \mathrm{ppm}$ may be due to phenolic hydroxyl protons. The signals in the range of 3.3( $\partial$ ) ppm are due to the proton of $\mathrm{COCH}_{3}$ groups. The much downfield chemical shift for phenolic $-\mathrm{OH}$ indicates clearly the intermolecular hydrogen bonding of - $\mathrm{OH}$ group [17, 18]. The signal appeared in the region at $2.29(\partial) \mathrm{ppm}$ is due to the methylene proton of Ar- $\mathrm{CH}_{2}$ bridge [19].

\section{Thermogravimetric analysis of $2,4-\mathrm{DHAAF}$ polymer resin:}

Fig. 6 shows thermogravimetric analysis of 2,4-DHAAF polymer. In order to explore the thermal degradation study of polymer resin, the thermogram has been studied minutely. Thermogram of 2,4-DHAAF polymer resin depicts four step decomposition in the temperature range $50-600^{\circ} \mathrm{C}$. The first step is slow decomposition between $50-150^{\circ} \mathrm{C}$ corresponds to loss of water molecule $(4.13 \%$ found and $4.12 \%$ calculated). The second step decomposition start from $150-260^{\circ} \mathrm{C}$ corresponds to $19.72 \%$ calculated and $19.74 \%$ found which represent degradation of four hydroxyl group which is attach with aromatic ring. The third step which is in the range $260-450^{\circ} \mathrm{C}$ corresponds to $42.66 \%$ calculated and $42.67 \%$ found which represent the loss of two acetyl group along with one methylene group. The fourth step corresponds to loss of three aromatic ring along with two methylene groups $(100 \%$ calculated and $100 \%$ found) in the range of $450-600^{\circ} \mathrm{C}$. In the present investigation Sharp-Wentworth and freeman-Carroll methods have been used to determine the kinetic parameters of 2,4-DHAAF polymer resin. 


\section{Sharp-Wentworth method :}

Using the equation derived by Sharp-Wentworth [1],

$$
\log [(\mathrm{dc} / \mathrm{dT}) /(1-\mathrm{c})]=\log (\mathrm{A} / \beta)-[\mathrm{Ea} / 2.303 \mathrm{R}] .1 / \mathrm{T} \text {. }
$$

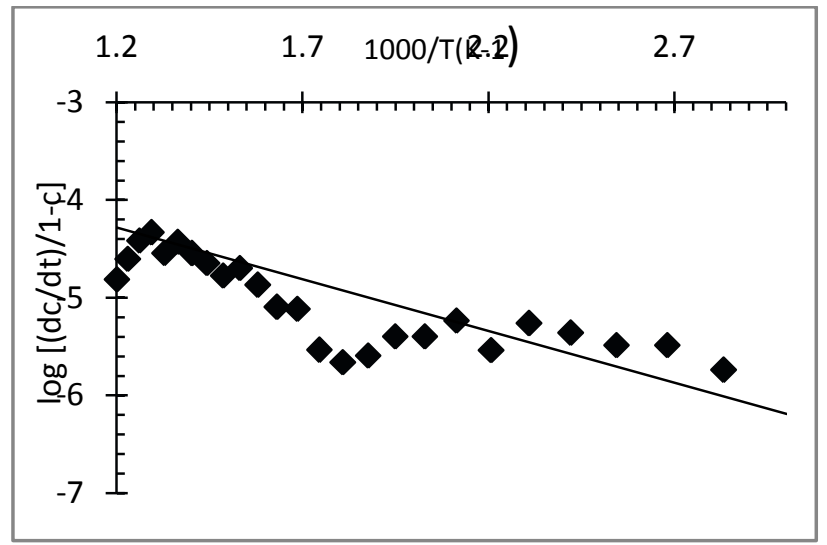

Fig 1: Sharp-Wentworth plot of 2,4-DHAAF polymer

By plotting the graph between $(\mathrm{dc} / \mathrm{dT}) /(1-\mathrm{c})$ vs $1 / \mathrm{T}$ we obtained the straight line which gave energy of activation ( Ea) from its slope. Where $\beta$ is the conversion at time $t, R$ is the gas constant and $T$ is the absolute temperature. The plots Fig. 1 give activation energies at different stages of degradation reaction take place.

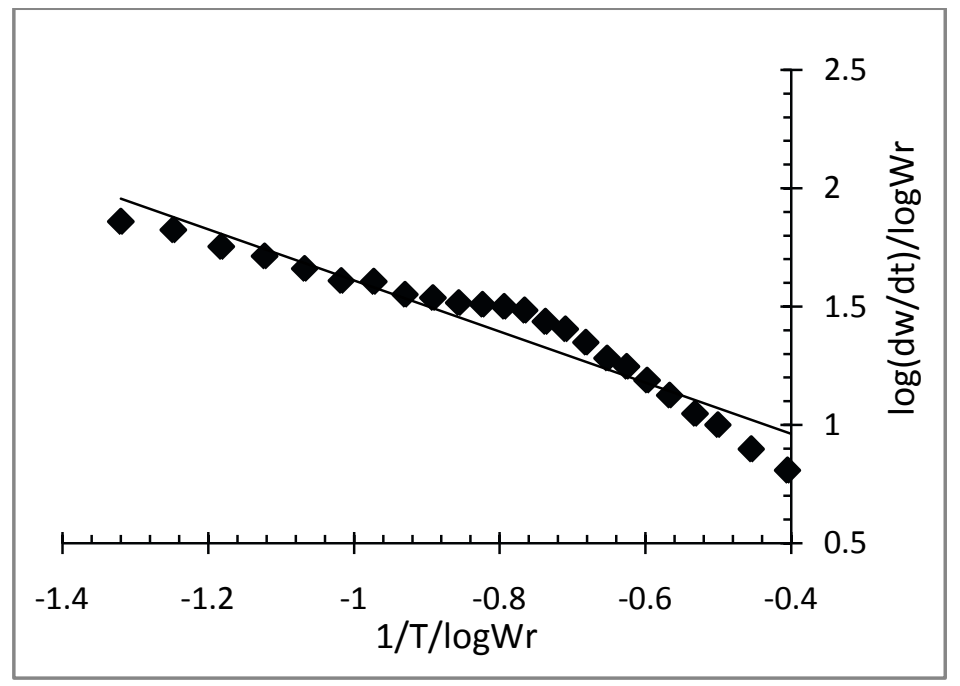

Fig 2: Freeman-Carroll plot of 2,4-DHAAF polymer 


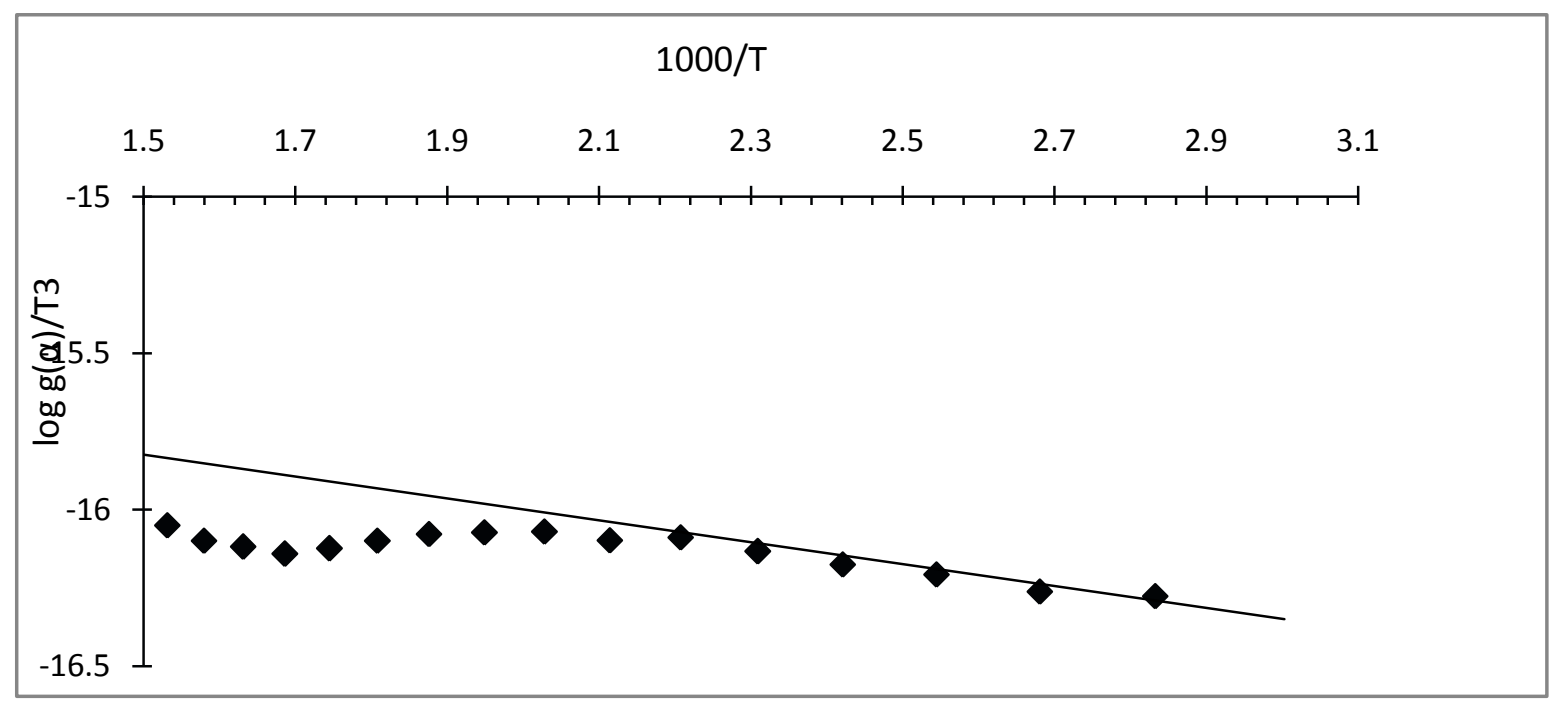

Fig. 3: Freeman-Carroll plot of 2,4-DHAAF polymer

A plot of percentage mass loss versus temperature is shown in Fig. 1 for 2,4-DHAAF polymer. From the TG curve, the thermo analytical and the decomposition temperature were determined (Table 1) to obtain the thermal stability of the polymer. The method described by Sharp Wentworth was adopted. Based on the initial decomposition temperature, the thermal stability of the polymer has been used here to define its thermal stability, neglecting the degree of decomposition (Table 1)

\section{Table 1: Results of Thermo gravimetric Analysis of 2,4-DHAAF copolymer resin}

\begin{tabular}{|c|c|c|c|c|c|c|c|}
\hline $\begin{array}{c}\text { Copolyme } \\
\text { r } \\
\text { Resin }\end{array}$ & $\begin{array}{c}\text { Half } \\
\text { decompositio } \\
\mathrm{n} \\
\text { temp. } \\
(\mathrm{K})\end{array}$ & $\begin{array}{c}\begin{array}{c}\text { Activatio } \\
n \\
\text { energy } \\
(\mathrm{KJ} / \mathrm{mol})\end{array} \\
\mathrm{FC} \\
\mathrm{SW}\end{array}$ & $\begin{array}{c}\text { Entrop } \\
\mathrm{y} \\
\text { change } \\
\square \mathrm{S} \\
\text { (J) }\end{array}$ & $\begin{array}{c}\text { Free } \\
\text { energy } \\
\text { chang } \\
\text { e } \\
\square \mathrm{F} \\
(\mathrm{KJ})\end{array}$ & $\begin{array}{c}\text { Frequenc } \\
\mathrm{y} \\
\text { factor } Z \\
\left(\mathrm{sec}^{-1}\right)\end{array}$ & $\begin{array}{c}\text { Apparen } \\
\mathrm{t} \\
\text { entropy } \\
\mathrm{S}^{*} \\
(\mathrm{~J})\end{array}$ & $\begin{array}{l}\text { Order } \\
\text { of } \\
\text { reactio } \\
n \\
\text { found } \\
\text { (n) }\end{array}$ \\
\hline $\begin{array}{c}2,4- \\
\text { DHAAF }\end{array}$ & 423 & $\begin{array}{l}20.50 \\
20.60\end{array}$ & $\begin{array}{c}- \\
302.53\end{array}$ & $\begin{array}{c}193.4 \\
5\end{array}$ & 352 & -13.78 & 0.99 \\
\hline
\end{tabular}


Using thermal decomposition data and then applying the SharpWentworth method Fig.2, activation energy is calculated which is in agreement with the activation energy calculated by Freeman-Carroll method, Fig. 8 [21]. Thermal activation energy plot of Sharp-Wentworth method and Freeman-Carroll method (Fig. 8) for the polymer have been shown. Thermodynamic parameters such as entropy change $(\square \mathrm{S})$, free energy change $(\square \mathrm{F})$, frequency factor $(Z)$ and Apparent entropy $\left(\mathrm{S}^{*}\right)$ calculated on the basis of thermal activation energy are given in Table 1.

By using the data of the Freeman-Carroll method, various thermodynamics parameters have been calculated (Table 1). From the abnormally low values of frequency factor, it may be concluded that the decomposition reaction of 2,4-DHAAF polymer can be classed as a 'slow 'reaction. There is no other obvious reason. Fairly good straight line plots are obtained using the two methods. This is expected since the decomposition of polymer is known not to obey first order kinetics perfectly.

\section{Chang method:}

Chang provides the following expression for thermal degradation kinetic studies

$\ln \frac{d \alpha d T}{\left(1-\alpha^{2}\right.}=\operatorname{og}(z)-\frac{E a}{R} \cdot \frac{1}{T}$

A plot of $[\ln (d a) / d t) /(1-a) n]$ vs $1 / T$ will yield a straight line if the order of decomposition reaction $\mathrm{n}$ is selected correctly. The slope of this line provides the $(-\mathrm{Ea} / \mathrm{R})$ values, from which Ea can be obtained. The plots give the activation energies of degradation reaction takes place.

\section{Coats - Redfern method:}

Coats and Redfern provides an approximation. This is an integral form of the rate equation. The simplified form of the equation is 
$\ln \frac{g(\propto)}{T^{2}}=\ln \frac{A R\left(1-2 R T / E_{a}\right)}{\emptyset E_{a}}-\frac{E_{a}}{R T}$

Where, $\mathrm{T}$ is Temperature, Ais Pre-exponential term, $\mathrm{R}$ is Gas constant, Ea is Energy of activation, $\phi$ is Heating rate and a is given by

$$
\propto=\frac{W_{0}-W_{t}}{W_{0}-W_{f}}
$$

Where, $\mathrm{W}_{0}$ is Initial weight of the sample, $\mathrm{W}_{\mathrm{t}}$ is Residual weight of the sample at the temperature, $\mathrm{W}_{\mathrm{f}}=$ Final weight of the sample and $\mathrm{g}(\mathrm{a})$ is equal to $-\ln (1-\mathrm{a})$ for $\mathrm{n}=1$ and $\left(\left[1-(1-\mathrm{a})^{\mathrm{n}}\right] /(1-\mathrm{n})\right)$ for $\mathrm{n} \# 1$. Thus a plot of either $\left(\left[1-(1-a)^{1-n}\right] / T^{2}(1-n)\right)$ vs $(1 / T)$ or $\left[-\ln (1-a) / T^{2}\right]$ vs $(1 / T)$ should result in a straight line of slope $\left[-E_{a} / R\right]$ for correct value of $n$, since it may be shown that for most value of $\mathrm{E}_{\mathrm{a}}$ and for the temperature range over which reaction generally occurs the expression $\ln \frac{A R\left(1-2 R T / E_{a}\right)}{\varnothing E_{a}}$ is constant.

This isoconversional (model-free) kinetic methods use to check the variation of the apparent activation energy values with degree of degradation. This kinetic analysis should be a starting point for obtain the useful information on the behavior of the sample.

\section{CONCLUSIONS}

A polymer 2,4-DHAAF based on the polycondesation reaction of 2Hydroxyacetophenone and catechol with formaldehyde in the presence of $2 \mathrm{M} \mathrm{HCl}$ as acid catalyst in 2:1:3 molar ratio. Thermogram of 2,4-DHAAF polymer resin shows activation energy calculated by the Freeman Carroll and Sharp-Wentworth methods are in good agreement with each other. Due to abnormally low value of frequency factor [Z] it may be classified as a slow reaction and no other obvious reason can be given. The value of entropy $[\Delta \mathrm{S}]$ indicates that the activated polymer has more ordered structure than the reactants and the reaction are slower than normal. It is very difficult to draw any unique conclusion from the 
magnitude of thermal activation energy [Ea]. Fairly straight -line plots are obtained using the two methods. Isoconversional of analysis of TGA plot indicate variation in activation energy of degradation process. The resins undergoes degradation at high temperature, indicates that the polymer resins under study are thermally stable at elevated temperature. The proposed structure of the 2,4-DHAAF polymer resin has been determined from the FTIR and ${ }^{1} \mathrm{H}$ NMR spectral studies.

\section{REFERENCES}

RM Silverstein, GC Bassler, and TC Morrill, (1991) Spectrometric Identification of organic compound, 5th Edi. John Wiley and Sons. Inc. Priented in Singapore.

PK Rahangdale, WB Gurnule, LJ Paliwal, and RB Kharat, (2003) Synth. React. Inorg. Met. Org. Chem., 33(7), 1187-1205.

RK Samal, BK Senapati, and TB Behuray, (1996) J. Appl. Polym. Sci., $62,655-660$

BS Furniss, AJ Hannaford, PWG Smith, and AR Tatchell, (1998) "Vogel's Text Book of Practical Organic Chemistry, Addison Westey Longman Ltd. England, First ISE Reprint.

RT Morrison, and RN Boyd, (1996 ) "Organic Chemistry, Sixth Edition, Prentice Hall of India Pvt. Ltd., New Delhi.

G Barth Howard, and W Mays Jimmy, (1991) "Modern Methods of Polymer Characterization," A Wiley Interscience Publication, John Willey and Sons, New York, 228.

A S Aswar, R G Mahale, P R Kakde, and S G Bhadange, (1998) J. Ind. Chem. Soc., 75:395.

NA Nadia Ahmed Mohamed, and AO Abeer Obaid Hamad Al Dossary, (2003) Polymer Degradation and Stability, 79(1), 6175.

PK Rahangdale, LJ Paliwal, and R B Kharat, (2000) Transition of the SAEST., 35 (1), 16-18. 